In der Rubrik „Literatur kompakt" werden die wichtigsten Originalarbeiten aus der internationalen Fachliteratur referiert.

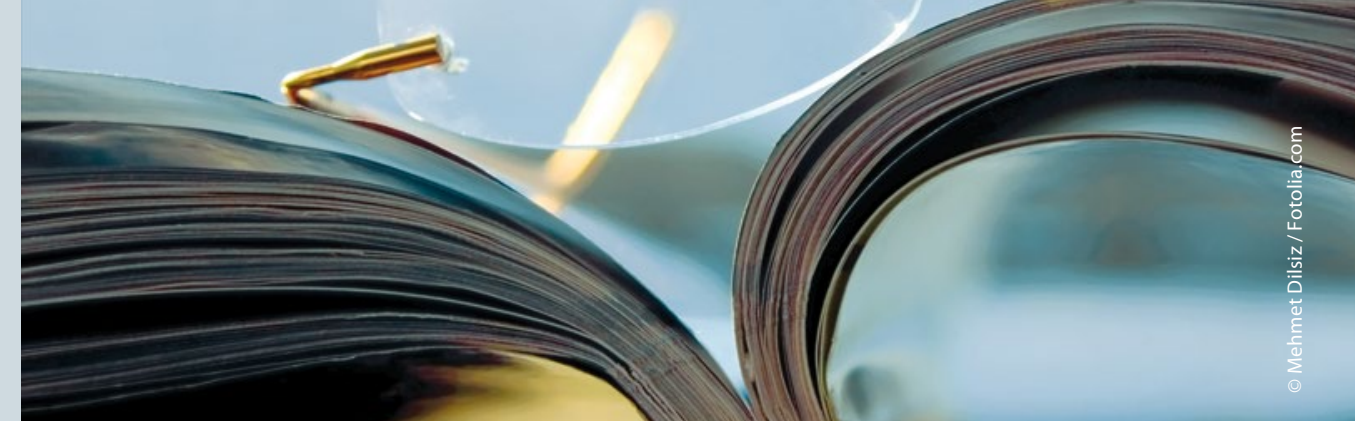

\section{Kombination von Simvastatin und Vitamin D3 reduziert die Zahl der Migränetage}

In einer placebokontrollierten Studie sollte geprüft werden, ob die Kombination von Simvastatin und Vitamin D3 in der Migräneprophylaxe wirksam ist.

$D^{\prime \prime}$ ie bisherige medikamentöse Prophylaxe der Migräne ist dadurch gekennzeichnet, dass viele der Substanzen unerwünschte Nebenwirkungen haben und deshalb die Compliance nicht besonders hoch ist. Die Arbeitsgruppe um Burstein in Boston hatte tierexperimentell belegt, dass Statine pleiotrope Effekte haben und die endotheliale Funktion verbessern. In einer populationsbezogenen Studie hatten sie herausgefunden, dass möglicherweise die Kombination von Statinen und Vitamin D die Migränefrequenz reduzieren kann. Deshalb führten sie jetzt eine randomisierte doppelblinde placebokontrollierte Studie durch.

Es handelt sich um eine Studie mit einer zwölfwöchigen Baseline-Phase und einer Behandlungsperiode von 24 Wochen. Es wurden 57 Erwachsene mit episodischer Migräne eingeschlossen. Die Studienteilnehmer erhielten zweimal $20 \mathrm{mg}$ Simvastatin pro Tag plus 1.000 IE Vitamin D3 zweimal täglich oder Placebo. Einschlusskriterium war eine Migränehäufigkeit von mehr als vier Tagen pro Monat. Der primäre Endpunkt war die Änderung der Migränetage zwischen der Basline-Periode und den Wochen 1 bis 12 und 13 bis 24 . Außerdem wurde die Responderrate berechnet, das heißt der Prozentsatz der Patienten mit einer über $50 \%$ igen Reduktion der Migränetage.
28 Teilnehmer erhielten die aktive Behandlung und 29 Placebo. Das mittlere Alter betrug 40 Jahre in der aktiven Behandlungsgruppe und 28 Jahre in der Placebogruppe. Über 90 \% der Teilnehmer waren Frauen. In der zwölfwöchigen Baseline-Phase hatten die Patienten in der aktiven Behandlungsgruppe im Schnitt 25 Kopfschmerztage und in der Placebogruppe 18.

In den ersten zwölf Wochen kam es in der aktiven Behandlungsgruppe zu einer Reduktion von durchschnittlich acht Migränetagen und in der zweiten zwölfwöchigen Behandlungsphase von neun Tagen. In der Placebogruppe kam es im ersten Abschnitt zu einer durchschnittlichen Zunahme von einem Migränetag und in der zweiten Behandlungsphase von drei Migränetagen. Diese Unterschiede waren mit $\mathrm{p}$-Werten von $<0,001$ signifikant. Die Responderrate betrug $25 \%$ und $29 \%$ in der ersten und zweiten Behandlungsphase mit Verum und 3\% in der Placebogruppe. Bei den Nebenwirkungen ergaben sich keine Unterschiede.

Fazit: Möglicherweise führt die Kombination von Simvastatin und Vitamin D3 zu einer Reduktion von Migränetagen in der Migräneprophylaxe.

Buettner C, Nir RR, Bertisch SM et al. Simvastatin and vitamin $D$ for migraine prevention: A randomized controlled trial. Ann Neurol 2015; 78(6): 970-81
Kommentar von Prof. Dr. med. Hans-Christoph Diener:

Diese prospektive randomisierte Studie scheint auf den ersten Blick zu belegen, dass die Kombination von Simvastatin und Vitamin D in der Migräneprophylaxe wirksam ist. Die Studie selbst ist technisch gut durchgeführt und hat eine lange Baselineund Beobachtungsphase. Allerdings ist die Zahl der Patienten sehr gering und es zeigten sich auch signifikante Unterschiede in bestimmten Charakteristika zum Zeitpunkt der Randomisierung.

Besonders auffällig bei den Studienergebnissen ist der fehlende Placeboeffekt. Es ist außerordentlich ungewöhnlich, dass in Studien zur Migräneprophylaxe Placebo nicht zu einer Reduktion der Migränehäufigkeit führt. Darüber hinaus kann die Studie nicht die wichtige Frage beantworten, welche der beiden Behandlungen möglicherweise die wirksamere Therapie darstellt.

Als ein großer Vorteil dieser Form der Migräneprophylaxe ist die Tatsache anzusehen, dass fast keine Nebenwirkungen auftraten. Bevor jetzt Simvastatin und Vitamin D3 als Therapeutika in die Migräneprophylaxe eingeführt werden, muss noch eine größere randomisierte Studie durchgeführt werden, um die in der hier vorliegenden Studie erhaltenen Ergebnisse zu replizieren.

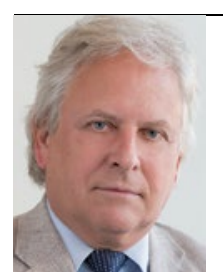

Prof. Dr. med. Hans-Christoph Diener Direktor der Klinik für Neurologie, Universitätsklinikum Essen, E-Mail: h.diener@uni-essen.de 